\title{
Existence of nonconstant periodic solutions for $p(t)$-Laplacian Hamiltonian system
}

Yuanfang $\mathrm{Ru}^{1,2^{*}}$ and Fanglei Wang ${ }^{1}$

${ }^{*}$ Correspondence:
ruyanfangmm@163.com
${ }^{1}$ College of Science, Hohai
University, Nanjing, P.R. China
${ }^{2}$ College of Science, China
Pharmaceutical University, Nanjing,
P.R. China

P.R. China

\begin{abstract}
The purpose of this paper is to consider the existence of periodic solutions for the $p(t)$-Laplacian Hamiltonian system. Some results are obtained by using the least action principle and the minimax methods.
\end{abstract}

Keywords: $p(t)$-Laplacian; Minimax principle; Least action principle; Periodic solution; Critical point

\section{Introduction}

In this paper, we consider the following problem:

$$
\left\{\begin{array}{l}
\frac{d}{d t}\left(|\dot{u}(t)|^{p(t)-2} \dot{u}(t)\right)-\nabla F(t, u(t))=0, \quad t \in[0, T] \\
u(0)-u(T)=\dot{u}(0)-\dot{u}(T)=0
\end{array}\right.
$$

where $T>0, u \in R^{N} . F(t, u)$ and $p(t)$ satisfy the following conditions:

$\left(F_{0}\right) F:[0, T] \times R^{N} \rightarrow R$ is measurable and $T$-periodic in $t$ for each $u \in R^{N}$ and continuously differentiable in $u$ for a.e. $t \in[0, T]$, and there exist $a \in C\left(R^{+}, R^{+}\right)$and $b \in L^{1}\left([0, T], R^{+}\right)$such that

$$
|F(t, u)| \leq a(|u|) b(t), \quad|\nabla F(t, u)| \leq a(|u|) b(t)
$$

for all $u \in R^{N}$ and a.e. $t \in[0, T]$.

(P) $p(t) \in C\left([0, T], R^{+}\right), p(t)=p(t+T)$ and

$$
1<p^{-}:=\min p(t) \leq p^{+}:=\max p(t)<+\infty
$$

The nonlinear problems involving the $p(t)$-Laplace type operator are extremely attractive because they can be used to model dynamical phenomena which arise from the study of elastic mechanics. The detailed application backgrounds of the $p(x)$-Laplace type operators can be found in $[1,2]$ and the references therein. The $p(t)$-Laplacian system possesses more complicated nonlinearity than that of the p-Laplacian, for example, it is not homogeneous, this causes many troubles, and some classical theories and methods, such as the theory of Sobolev spaces, are not applicable.

(c) The Author(s) 2019. This article is distributed under the terms of the Creative Commons Attribution 4.0 International License (http://creativecommons.org/licenses/by/4.0/), which permits unrestricted use, distribution, and reproduction in any medium, provided you give appropriate credit to the original author(s) and the source, provide a link to the Creative Commons license, and indicate if changes were made. 
In recent years, many researchers studied the periodic solutions of the system (1.1). Some existence results are obtained by using the least action principle and minimax methods in critical point theory. Many solvability conditions were given, such as the coercivity condition, the periodicity condition, the convexity condition, the boundedness condition, the subadditive condition and the sublinear condition. We refer to [3-13].

When $F(t, x)=G(x)+H(t, x)$, the $p(t)$-Laplacian system has also been studied by many authors. Especially, in [13], the authors supposed that $H(t, x)$ is $p^{-}$-sublinear, that is, there exist $f, g \in L^{1}\left([0, T], R^{+}\right)$and $\alpha \in\left[0, p^{-}\right)$such that

$$
|\nabla H(t, x)| \leq f(t)|x|^{\alpha}+g(t)
$$

for all $x \in R^{N}$ and a.e. $t \in[0, T]$, and there exist $0 \leq r<1 /\left(p^{+} T^{p^{-}}\right)$and $1 \leq \beta \leq p^{-}$such that

$$
\left\langle\nabla G(x)-\nabla G(y), x-y|\geq-r| x-\left.y\right|^{\beta}\right.
$$

for all $x, y \in R^{N}$.

Moreover, they consider system (1.1) with $F(t, x)$ which is the sum of a subconvex function and another function under suitable conditions, by the least action principle and the saddle point theorem, they obtain some existence results.

Motivated by the results mentioned above, we aim in this paper to study the system (1.1) with a potential $F(t, x)$ which is also the sum of $F_{1}(t, x)$ and $F(x)$, where the conditions on $F_{1}(t, x)$ and $F(x)$ are more general and simple. By the least action principle, we obtained three existence results.

This paper is organized as follows. In Sect. 2, we give some necessary preliminary knowledge on variable exponent Sobolev spaces. In Sect. 3, we present our main results and completed the proof. In Sect. 4, we give some examples to illustrate our results.

\section{Preliminary}

For the convenience of readers, we first state some properties of the variable exponent Lebesgue-Sobolev spaces $L^{p(t)}$ and $W_{T}^{1, p(t)}$ (for details, see [14-18]). In the following, we use $|\cdot|$ to denote the Euclidean norm in $R^{N}$.

Let $p(t)$ satisfy the condition $(P)$ and define a generalized Lebesgue space

$$
L^{p(t)}\left([0, T] ; R^{N}\right)=\left\{u \in L^{1}\left([0, T] ; R^{N}\right): \int_{0}^{T}|u|^{p(t)} d t<\infty\right\},
$$

with the norm

$$
|u|_{L^{p(t)}}=|u|_{p(t)}=\inf \left\{\lambda>0: \int_{0}^{T}\left|\frac{u}{\lambda}\right|^{p(t)} d t \leq 1\right\} .
$$

Define

$$
\left.C_{T}^{\infty}=C_{T}^{\infty}\left(R ; R^{N}\right)=\left\{u \in C^{\infty}\left(R ; R^{N}\right)\right): u \text { is } T \text {-periodic }\right\} .
$$

For $u, v \in L^{1}\left([0, T] ; \mathbb{R}^{N}\right)$, if

$$
\int_{0}^{T}\left(u(t), \varphi^{\prime}(t)\right) d t=-\int_{0}^{T}(v(t), \varphi(t)) d t, \quad \forall \varphi \in C_{T}^{\infty},
$$

then $v(t)$ is called a $T$-weak derivative of $u(t)$ and is denoted by $\dot{u}(t)$. 
It has been proved that (see [19], page 6)

$$
\int_{0}^{T} \dot{u}(s) d s=0,
$$

and there exists $C \in \mathbb{R}^{N}$ such that

$$
u(t)=\int_{0}^{T} v(s) d s+C, \quad \text { a.e. } t \in[0, T]
$$

and $u(0)=u(T)=C$.

Define a generalized Sobolev space

$$
W_{T}^{1, p(t)}\left([0, T] ; R^{N}\right)=\left\{u \in L^{p(t)}\left([0, T] ; R^{N}\right): \dot{u} \in L^{p(t)}\left([0, T] ; R^{N}\right)\right\}
$$

with the norm

$$
\|u\|_{W_{T}^{1, p(t)}}=\|u\|=|u|_{p(t)}+|\dot{u}|_{p(t)} .
$$

For $u \in W_{T}^{1, p(t)}\left([0, T] ; R^{N}\right)$, let

$$
\bar{u}=\frac{1}{T} \int_{0}^{T} u(s) d s, \quad \tilde{u}(t)=u(t)-\bar{u},
$$

and

$$
\widetilde{W}_{T}^{1, p(t)}\left([0, T] ; R^{N}\right)=\left\{u \in W_{T}^{1, p(t)}\left([0, T] ; R^{N}\right): \int_{0}^{T} u(s) d s=0\right\},
$$

then

$$
W_{T}^{1, p(t)}\left([0, T] ; R^{N}\right)=\widetilde{W}_{T}^{1, p(t)}\left([0, T] ; R^{N}\right) \oplus R^{N} .
$$

In the following we use $L^{p(t)}, W_{T}^{1, p(t)}, \widetilde{W}_{T}^{1, p(t)}$ to denote the $L^{p(t)}\left([0, T] ; R^{N}\right), W_{T}^{1, p(t)}([0, T]$; $\left.R^{N}\right), \widetilde{W}_{T}^{1, p(t)}\left([0, T] ; R^{N}\right)$, respectively.

Lemma 2.1 ([20]) There is a continuous embedding $W^{1, p(t)} \hookrightarrow C\left([0, T], \mathbb{R}^{N}\right)$, when $p^{-}>1$, the embedding is compact. And for $u \in \widetilde{W}_{T}^{1, p(t)}$, there is a constant $C$ independent of $u$ such that

$$
\|u\|_{\infty} \leq C\|u\|
$$

Lemma $2.2([2,20])$ For every $u \in \widetilde{W}_{T}^{1, p(t)}$, there exist constants $c_{1}^{\prime}, c_{1}$ such that

$$
\begin{aligned}
\|u\|_{\infty} & \leq c_{1}|\dot{u}|_{p(t)}, \\
\|u\|_{\infty} & \leq 2 c_{1}^{\prime}\left[\left(\int_{0}^{T}|\dot{u}(t)|^{p(t)} d t\right)^{\frac{1}{p^{-}}}+T^{\frac{1}{p^{-}}}\right]
\end{aligned}
$$

where $c_{1}, c_{1}^{\prime}>0$. 
Lemma 2.3 ([20]) Let $u=\bar{u}+\tilde{u} \in W_{T}^{1, p(t)}$, then the norm $|\widetilde{\dot{u}}|_{p(t)}$ is an equivalent norm on $\widetilde{W}_{T}^{1, p(t)}$ and $|\bar{u}|+|\dot{u}|_{p(t)}$ is an equivalent norm on $W_{T}^{1, p(t)}$. Therefore, for $u \in W_{T}^{1, p(t)}$,

$$
\|u\| \rightarrow \infty \quad \Rightarrow \quad|\bar{u}|+\int_{0}^{T}\left|u^{\prime}(t)\right|^{p(t)} d t \rightarrow \infty
$$

Lemma 2.4 ([2])

(i) The space $\left(L^{p(t)},|\cdot|\right)$ is a separable, reflexive, uniform convex Banach space, and its conjugate space is $L^{q(t)}$, where $\frac{1}{p(t)}+\frac{1}{q(t)}$. For any $u \in L^{p(t)}$ and $v \in L^{q(t)}$, we have

$$
\left|\int_{0}^{T} u(t) v(t) d t\right| \leq 2|u|_{p(t)}|v|_{q(t)}
$$

(ii) If $p_{1}(t), p_{2}(t) \in C\left([0, T], \mathbb{R}^{1}\right)$ and $1<p_{1}(t) \leq p_{2}(t)$ for any $t \in[0, T]$, then $L^{p_{2}(t)} \hookrightarrow L^{p_{1}(t)}$, and the embedding is continuous.

\section{Main results of problem (1.1)}

Definition 3.1 A function $u(t) \in W_{T}^{1, p(t)}$ is called a weak solution of (1.1), if $|\dot{u}(t)|^{p(t)-2} \dot{u}(t)$ has a weak derivative, still denoted by $\frac{d}{d t}\left(\left.\dot{u}(t)\right|^{p(t)-2} \dot{u}(t)\right)$, such that

$$
\left\{\begin{array}{l}
\frac{d}{d t}\left(|\dot{u}(t)|^{p(t)-2} \dot{u}(t)\right)-\nabla F(t, u(t))=0, \quad \text { a.e. } t \in[0, T] \\
u(0)-u(T)=\dot{u}(0)-\dot{u}(T)=0 .
\end{array}\right.
$$

Define a functional $\varphi$ on $W_{T}^{1, p(t)}$ by

$$
\left.\varphi(u)=\int_{0}^{T} \frac{1}{p(t)}\right)|\dot{u}(t)|^{p(t)} d t+\int_{0}^{T} F(t, u(t)) d t
$$

Lemma 3.1 Suppose that assumptions $\left(F_{0}\right)$ and $(P)$ hold, then the functional $\varphi$ is continuously differentiable on $W_{T}^{1, p(t)}$ and

$$
\left\langle\varphi^{\prime}(u), v\right\rangle=\int_{0}^{T}\left(|\dot{u}(t)|^{p(t)-2} \dot{u}(t), \dot{v}(t)\right) d t+\int_{0}^{T}(\nabla F(t, u(t)), v(t)) d t
$$

for all $u, v \in W_{T}^{1, p(t)}$. And if there exists $u \in W_{T}^{1, p(t)}$ such that $\left\langle\varphi^{\prime}(u), v\right\rangle=0$ for all $v \in W_{T}^{1, p(t)}$, then $u$ is a weak solution (1.1).

Proof For convenience, define functionals $J$ and $H$ on $W_{T}^{1, p(t)}$ by

$$
\left.J(u)=\int_{0}^{T} \frac{1}{p(t)}\right)|\dot{u}(t)|^{p(t)} d t, \quad H(u)=\int_{0}^{T} F(t, u(t)) d t .
$$

It is easy to see that $([18])$

$$
\varphi(u)=J(u)+H(u), \quad\left\langle\varphi^{\prime}(u), v\right\rangle=\left\langle J^{\prime}(u), v\right\rangle+\left\langle H^{\prime}(u), v\right\rangle,
$$

for all $v \in W_{T}^{1, p(t)}$ and

$$
\left\langle J^{\prime}(u), v\right\rangle=\int_{0}^{T}\left(|\dot{u}(t)|^{p(t)-2} \dot{u}(t), \dot{v}(t)\right) d t
$$


It is easy to see that

$$
\left\langle H^{\prime}(u), v\right\rangle=\int_{0}^{T}(\nabla F(t, u(t)), v(t)) d t .
$$

In fact

$$
\begin{aligned}
\frac{1}{s}[H(u+s v)-H(u)] & =\frac{1}{s} \int_{0}^{T}[F(t, u(t)+s v(t))-F(t, u(t))] d t \\
& =\int_{0}^{T}(\nabla F(t, u(t)+\theta s v(t)), v(t)) d t
\end{aligned}
$$

where $0<\theta<1$.

Since

$$
|\nabla F(t, u(t)+\theta s v(t))| \leq a(|u(t)+\theta s v(t)|) b(t) \leq a_{0} b(t)
$$

and $\nabla F(t, u(t)+\theta s v(t)) \rightarrow \nabla F(t, u(t))$, a.e. $t \in[0, T]$, as $s \rightarrow 0$.

Here $a_{0}$ is some constant.

By the Lebegue dominated convergence theorem, we have

$$
\lim _{s \rightarrow 0} \frac{1}{s}[H(u+s v)-H(u)]=\int_{0}^{T}(\nabla F(t, u(t), v(t)) d t,
$$

which implies that $H$ is Gâteaux differentiable, and

$$
\left\langle H^{\prime}(u), v\right\rangle=\int_{0}^{T}(\nabla F(t, u(t)), v(t)) d t, \forall v \in W_{T}^{1, p(t)}
$$

Next we will prove that the functional $\varphi$ is continuous differentiable, it suffices to show that both of $J^{\prime}$ and $H^{\prime}$ are continuous on $W_{T}^{1, p(t)}$.

Let $u_{n}, u \in W_{T}^{1, p(t)}$, such that $\left\|u_{n}-u\right\| \rightarrow 0(n \rightarrow \infty)$. Obviously,

$$
\left\|u_{n}-u\right\| \rightarrow 0 \quad \Leftrightarrow \quad\left|u_{n}-u\right|_{p(t)} \rightarrow 0 \quad \text { and } \quad\left|u_{n}^{\prime}-u^{\prime}\right|_{p(t)} \rightarrow 0
$$

Note that the mapping defined by $u \in L^{p(t)} \rightarrow|u(t)|^{p(t)-2} u \in L^{q(t)}$, where $\frac{1}{p(t)}+\frac{1}{q(t)}=1$, is a bounded and continuous mapping from $L^{p(t)}$ into $L^{q(t)}$ (see [15]). It follows from (3.1) that

$$
\left.2|| u_{n}^{\prime}\right|^{p(t)-2} u_{n}^{\prime}-\left.\left|u^{\prime}\right|^{p(t)-2} u^{\prime}\right|_{q(t)} \rightarrow 0
$$

which, combined with Lemma 2.4, shows

$$
\begin{aligned}
\left\|J^{\prime}\left(u_{n}\right)-J^{\prime}(u)\right\| & =\sup _{\|v\| \leq 1}\left|\int_{0}^{T}\left(\left|u_{n}^{\prime}\right|^{p(t)-2} u_{n}^{\prime}-\left|u^{\prime}\right|^{p(t)-2} u^{\prime}, v^{\prime}(t)\right)\right| \\
& \leq 2 \sup _{\|v\| \leq 1} \|\left. u_{n}^{\prime}\right|^{p(t)-2} u_{n}^{\prime}-\left.\left|u^{\prime}\right|^{p(t)-2} u^{\prime}\right|_{q(t)}\left|v^{\prime}\right|_{p(t)} \\
& \leq\left. 2|| u_{n}^{\prime}\right|^{p(t)-2} u_{n}^{\prime}-\left.\left|u^{\prime}\right|^{p(t)-2} u^{\prime}\right|_{q(t)} \rightarrow 0 .
\end{aligned}
$$

Hence, $J^{\prime}$ is continuous. 
Next, we prove the continuity of $H^{\prime}(u): W_{T}^{1, p(t)} \rightarrow\left(W_{T}^{1, p(t)}\right)^{*}$.

Let $u_{n}, u \in W_{T}^{1, p(t)}$, such that $\left\|u_{n}-u\right\| \rightarrow 0(n \rightarrow \infty)$.

From Lemma 2.1, it follows that

$$
u_{n}, u \in C\left([0, T], \mathbb{R}^{N}\right) \quad \text { and } \max _{0 \leq t \leq T}\left|u_{n}(t)-u(t)\right| \rightarrow 0 \quad(n \rightarrow \infty) .
$$

From the condition $\left(F_{0}\right)$, we have

$$
\left|\nabla F\left(t, u_{n}(t)\right)-\nabla F(t, u(t))\right| \rightarrow 0 \quad(n \rightarrow \infty) \text {, a.e. } t \in[0, T],
$$

and $a\left(\left|u_{n}(t)\right|\right) \rightarrow a(|u(t)|)$ in $C\left([0, T] ; \mathbb{R}^{N}\right)$.

Therefore, for $n$ large enough,

$$
a\left(\left|u_{n}(t)\right|\right) \leq a(|u(t)|)+1
$$

Then we have

$$
\begin{aligned}
\left|\nabla F\left(t, u_{n}(t)\right)-\nabla F(t, u(t))\right| & \leq\left(a(|u(t)|)+a\left(\left|u_{n}(t)\right|\right)\right) b(t) \\
& \leq\left(2 \max _{0 \leq t \leq T} a(|u(t)|)+1\right) b(t) \\
& \leq C b(t) .
\end{aligned}
$$

Here $C$ is some constant.

Then from (3.2) and (3.3), together with the Lebesgue dominated convergence theorem, we get

$$
\int_{0}^{T}\left|\nabla F\left(t, u_{n}(t)\right)-\nabla F(t, u(t))\right| d t \rightarrow 0 \quad(n \rightarrow \infty) .
$$

Therefore,

$$
\begin{aligned}
& \left\|H^{\prime}\left(u_{n}\right)-H^{\prime}(u)\right\|_{\left(W_{T}^{1, p(t)}\right)^{*}} \\
& =\sup _{\|v\| \leq 1, v \in W_{T}^{1, p(t)}}\left|\int_{0}^{T}\left(\nabla F\left(t, u_{n}(t)\right)-\nabla F(t, u(t)), v(t)\right) d t\right| \\
& \leq \sup _{\|v\| \leq 1, v \in W_{T}^{1, p(t)}} \int_{0}^{T}\left|\nabla F\left(t, u_{n}(t)\right)-\nabla F(t, u(t))\right| \cdot|v(t)| d t \\
& \leq C \int_{0}^{T}\left|\nabla F\left(t, u_{n}(t)\right)-\nabla F(t, u(t))\right| d t \rightarrow 0 \quad(n \rightarrow \infty) .
\end{aligned}
$$

So $H^{\prime}$ is continuous.

Suppose that $u \in W_{T}^{1, p(t)}$ such that

$$
\left\langle\varphi^{\prime}(u), v\right\rangle=0, \quad \forall v \in W_{T}^{1, p(t)},
$$


then

$$
\int_{0}^{T}\left(|\dot{u}(t)|^{p(t)-2} \dot{u}(t), v(t)\right) d t=-\int_{0}^{T}(\nabla F(t, u(t)), v(t)) d t
$$

for all $v \in W_{T}^{1, p(t)}$, obviously, also for all $v \in C_{T}^{\infty}$.

From $\left(F_{0}\right)$ and Lemma 2.1, $\nabla F(t, u(t)) \in L^{1}$, therefore, (3.4) implies that $\nabla F(t, u(t))$ is the weak derivative $\frac{d}{d t}\left(|\dot{u}(t)|^{p(t)-2} \dot{u}(t)\right)$ of $|\dot{u}(t)|^{p(t)-2} \dot{u}(t)$, and

$$
\begin{aligned}
& \left.\frac{d}{d t}\left(|\dot{u}(t)|^{p(t)-2} \dot{u}(t)\right)=\nabla F(t, u(t)), v(t)\right), \quad \text { a.e. } t \in[0, T], \\
& |\dot{u}(0)|^{p(0)-2} \dot{u}(0)=|\dot{u}(T)|^{p(T)-2} \dot{u}(T),
\end{aligned}
$$

since $p(0)=p(T)$, it follows that $\dot{u}(0)=\dot{u}(T)$, so $u(t)$ is the weak solution of (1.1).

The proof is completed.

For the reader's convenience, we give a definition.

Definition 3.2 ([21]) A function $F: R^{N} \rightarrow R$ is said to be $(\lambda, \mu)$-subconvex if

$$
F(\lambda(x+y)) \leq \mu(F(x)+F(y))
$$

for some $\lambda, \mu>0$ and all $x, y \in R^{N}$.

Theorem 3.1 Let $F(t, x)=F_{1}(t, x)+F_{2}(x)$, where $F_{1}$ and $F_{2}$ satisfy $\left(F_{0}\right)$ and the following conditions:

(i) $F_{1}(t, \cdot)$ is $\left(\lambda p^{-}, \mu p^{-}\right)$-subconvex for a.e. $t \in[0, T]$, where

$$
\lambda p^{-}>1, \quad 1<2 \mu p^{-}<\left(\lambda p^{-}\right)^{p^{-}}
$$

(ii) there exists a constant $0 \leq r<\frac{p^{-}}{\left(4 c_{1}\right)^{p^{-}} T p^{+}}$such that

$$
\left(\nabla F_{2}(x)-\nabla F_{2}(y), x-y\right) \geq-r|x-y|^{p^{-}}
$$

for all $x, y \in R^{N}$ and a.e. $t \in[0, T]$;

(iii)

$$
\frac{1}{\mu p^{-}} \int_{0}^{T} F_{1}\left(t, \lambda p^{-} x\right) d t+\int_{0}^{T} F_{2}(x) d t \rightarrow+\infty
$$

$$
\text { as }|x| \rightarrow+\infty .
$$

Then problem (1.1) has at least one nontrivial solution which minimizes $\varphi$ on $W_{T}^{1, p(t)}$.

Proof Let $\alpha=\log _{\lambda p^{-}}\left(2 \mu p^{-}\right)$, then $0<\alpha<p^{-}$. For $|x|>1$, there exists a positive integer $n$ such that

$$
n-1<\log _{\lambda p^{-}}|x| \leq n
$$


Ru and Wang Boundary Value Problems

(2019) 2019:135

Page 8 of 13

Furthermore, we have $|x|^{\alpha} \geq\left(\lambda p^{-}\right)^{(n-1) \alpha}=\left(2 \mu p^{-}\right)^{n-1}$ and $|x| \leq\left(\lambda p^{-}\right)^{n}$. Then from (i) and condition $\left(F_{0}\right)$, it follows that

$$
\begin{aligned}
F_{1}(t, x) & =F_{1}\left(t, \lambda p^{-}\left(\frac{x}{2 \lambda p^{-}}+\frac{x}{2 \lambda p^{-}}\right)\right) \leq 2 \mu p^{-} F_{1}\left(t, \frac{x}{2 \lambda p^{-}}\right) \leq \cdots \\
& \leq\left(2 \mu p^{-}\right)^{n} F_{1}\left(t, \frac{x}{\left(2 \lambda p^{-}\right)^{n}}\right) \leq 2 \mu p^{-}|x|^{\alpha} a_{0} b(t),
\end{aligned}
$$

for ace. $t \in[0, T]$ and all $|x|>1$, where $a_{0}=\max _{0 \leq s \leq 1} a(s)$.

Moreover, we have

$$
F_{1}(t, x) \leq\left(2 \mu p^{-}|x|^{\alpha}+1\right) a_{0} b(t)
$$

for ace. $t \in[0, T]$ and all $x \in R^{N}, 0<\alpha<p^{-}$.

By (i) and Lemma 2.2, it is obvious that

$$
\begin{aligned}
\int_{0}^{T} F_{1}(t, u(t)) d t & \geq \frac{1}{\mu p^{-}} \int_{0}^{T} F_{1}\left(t, \lambda p^{-} \bar{u}\right) d t-\int_{0}^{T} F_{1}(t,-\widetilde{u}) d t \\
& \geq \frac{1}{\mu p^{-}} \int_{0}^{T} F_{1}\left(t, \lambda p^{-} \bar{u}\right) d t-\left(2 \mu p^{-}\|\tilde{u}\|_{\infty}^{\alpha}+1\right) a_{0} \int_{0}^{T} b(t) d t \\
& \geq \frac{1}{\mu p^{-}} \int_{0}^{T} F_{1}\left(t, \lambda p^{-} \bar{u}\right) d t-c_{2}\left(\int_{0}^{T}|\dot{u}(t)|^{p(t)} d t\right)^{\frac{\alpha}{p^{-}}}-c_{3}
\end{aligned}
$$

for all $u \in W_{T}^{1, p(t)}$ and some constants $c_{2}, c_{3}$.

Using (ii) and Lemma 2.2, we have

$$
\begin{aligned}
\int_{0}^{T}\left(F_{2}(u)-F_{2}(\bar{u})\right) d t & =\int_{0}^{T} \int_{0}^{1}\left(\nabla F_{2}(\bar{u}+s \tilde{u}), \tilde{u}\right) d s d t \\
& =\int_{0}^{T} \int_{0}^{1} \frac{1}{s}\left(\nabla F_{2}(\bar{u}+s \tilde{u})-\nabla F_{2}(\bar{u}), s \tilde{u}\right) d s d t \\
& \geq-\int_{0}^{T} \int_{0}^{1} \frac{r}{s}|s \tilde{u}|^{p^{-}} d s d t \\
& =-r \int_{0}^{T} \frac{1}{p^{-}}|\widetilde{u}|^{p^{-}} d t \\
& \geq-\frac{r T}{p^{-}}\|\tilde{u}\|_{\infty}^{p^{-}} \\
& \geq-\frac{2^{2 p^{-}} r T c_{1}^{p^{-}}}{p^{-}} \int_{0}^{T}|\dot{u}|^{p(t)} d t+c_{4}
\end{aligned}
$$

for all $u \in W_{T}^{1, p(t)}$ and a constant $c_{4}$.

Combining (iii), (3.5) and (3.6), we get

$$
\begin{aligned}
\varphi(u) & \left.=\int_{0}^{T} \frac{1}{p(t)}\right)|\dot{u}(t)|^{p(t)} d t+\int_{0}^{T} F(t, u(t)) d t \\
& \geq \frac{1}{p^{+}} \int_{0}^{T}|\dot{u}(t)|^{p(t)} d t+\int_{0}^{T} F_{1}(t, u(t)) d t+\int_{0}^{T}\left(F_{2}(u)-F_{2}(\bar{u})\right) d t+\int_{0}^{T} F_{2}(\bar{u}) d t
\end{aligned}
$$




$$
\begin{aligned}
\geq & \left(\frac{1}{p^{+}}-\frac{\left(4 c_{1}\right)^{p^{-}} r T}{p^{-}}\right) \int_{0}^{T}|\dot{u}(t)|^{p(t)} d t+\left(\frac{1}{\mu p^{-}} \int_{0}^{T} F_{1}\left(t, \lambda p^{-} \bar{u}\right) d t+\int_{0}^{T} F_{2}(\bar{u}) d t\right) \\
& -c_{2}\left(\int_{0}^{T}|\dot{u}(t)|^{p(t)} d t\right)^{\frac{\alpha}{p^{-}}}+c_{5}
\end{aligned}
$$

for all $u \in W_{T}^{1, p(t)}$ and some constants $c_{2}, c_{5}$, it follows from (iii), $0<\alpha<p^{-}, 0 \leq r<\frac{p^{-}}{\left(4 c_{1}\right)^{p^{-}} T p^{+}}$ and Lemma 2.3, $\varphi(u) \rightarrow+\infty$ as $\|u\| \rightarrow+\infty$. Applying Theorem 1.1 and Corollary 1.1 in Mawhin-Willem [19], we complete the proof.

Theorem 3.2 Let $F(t, x)=F_{1}(t, x)+F_{2}(x)$, where $F_{1}$ and $F_{2}$ satisfy $\left(F_{0}\right)$ and the following conditions:

(i) there exist $f(t), m(t) \in L^{1}\left([0, T] ; R^{+}\right)$and $\gamma \in\left[0, p^{-}-1\right)$ such that

$$
\left|\nabla F_{1}(t, x)\right| \leq f(t)|x|^{\gamma}+m(t)
$$

for all $x \in R^{N}$ and a.e. $t \in[0, T]$;

(ii) there exist constants $0 \leq r<\frac{p^{-}}{p^{+} T\left(4 c_{1}\right)^{p^{-}}}$, such that

$$
\left(\nabla F_{2}(x)-\nabla F_{2}(y), x-y\right) \geq-r|x-y|^{p^{-}}
$$

for all $x, y \in R^{N}$ and a.e. $t \in[0, T]$

(iii)

$$
\begin{aligned}
& \liminf _{|x| \rightarrow \infty} \frac{1}{|x|^{\gamma q}} \int_{0}^{T} F(t, x) d t \geq A_{1} c(\varepsilon) \\
& \text { as }|x| \rightarrow+\infty \text {, where } \frac{1}{p^{-}}+\frac{1}{q}=1, A_{1}, c(\varepsilon) \text { are defined in the proof. }
\end{aligned}
$$

Then problem (1.1) has at least one nontrivial solution which minimizes $\varphi$ on $W_{T}^{1, p(t)}$.

Proof For convenience, we denote

$$
A_{1}=2^{\gamma} \int_{0}^{T} f(t) d t, \quad A_{2}=\int_{0}^{T} m(t) d t .
$$

By condition (i), the $\varepsilon$-Young inequality, Sobolev's inequality and Lemma 2.2, for any $u \in$ $W_{T}^{1, p(t)}$ and some constants $c_{6}, c_{7}, c_{8}$, we have

$$
\begin{aligned}
& \mid \int_{0}^{T}\left(F_{1}(t, u(t))-F_{1}(t, \bar{u}) d t \mid\right. \\
& \quad=\left|\int_{0}^{T} \int_{0}^{1}\left(\nabla F_{1}(t, \bar{u}+s \tilde{u}(t)), \tilde{u}(t)\right) d s d t\right| \\
& \quad \leq \int_{0}^{T} \int_{0}^{1} f(t)|\bar{u}+s \tilde{u}(t)|^{\gamma}|\tilde{u}(t)| d s d t+\int_{0}^{T} \int_{0}^{1} m(t)|\tilde{u}(t)| d s d t \\
& \quad \leq 2^{\gamma}\left(|\bar{u}|^{\gamma}+\|\tilde{u}\|_{\infty}^{\gamma}\right)\|\tilde{u}\|_{\infty} \int_{0}^{T} f(t) d t+\|\tilde{u}\|_{\infty} \int_{0}^{T} m(t) d t \\
& \quad=A_{1}|\bar{u}|^{\gamma} \cdot\|\tilde{u}\|_{\infty}+A_{1}\|\tilde{u}\|_{\infty}^{\gamma+1}+A_{2}\|\tilde{u}\|_{\infty} \\
& \quad \leq A_{1}\left(\varepsilon\|\tilde{u}\|_{\infty}^{p^{-}}+c(\varepsilon)|\bar{u}|^{\gamma q}\right)+c_{6}\left(\int_{0}^{T}|\dot{u}(t)|^{p(t)} d t\right)^{\frac{\gamma+1}{p^{-}}}+c_{7}\left(\int_{0}^{T}|\dot{u}(t)|^{p(t)} d t\right)^{\frac{1}{p^{-}}}+c_{8}
\end{aligned}
$$




$$
\begin{aligned}
\leq & A_{1} \varepsilon\left(4 c_{1}\right)^{p^{-}} \int_{0}^{T}|\dot{u}(t)|^{p(t)} d t+A_{1} c(\varepsilon)|\bar{u}|^{\gamma q} \\
& +c_{6}\left(\int_{0}^{T}|\dot{u}(t)|^{p(t)} d t\right)^{\frac{\gamma+1}{p^{-}}}+c_{7}\left(\int_{0}^{T}|\dot{u}(t)|^{p(t)} d t\right)^{\frac{1}{p^{-}}}+c_{8}
\end{aligned}
$$

where $\varepsilon$ satisfies $0<\varepsilon<\frac{1}{A_{1}}\left(\frac{1}{\left(4 c_{1}\right)^{p^{-}} p^{+}}-\frac{r T}{p^{-}}\right), c(\varepsilon)=\left(\varepsilon p^{-}\right)^{-\frac{q}{p^{-}}} \cdot \frac{1}{q}$.

Combining (3.6), (3.7) and (iii), we obtain

$$
\begin{aligned}
\varphi(u)= & \left.\int_{0}^{T} \frac{1}{p(t)}\right)|\dot{u}(t)|^{p(t)} d t+\int_{0}^{T} F(t, u(t)) d t \\
\geq & \frac{1}{p^{+}} \int_{0}^{T}|\dot{u}(t)|^{p(t)} d t+\int_{0}^{T}\left(F_{1}(t, u(t))-F_{1}(t, \bar{u})\right) d t \\
& +\int_{0}^{T}\left(F_{2}(u)-F_{2}(\bar{u})\right) d t+\int_{0}^{T} F(t, \bar{u}) d t \\
\geq & \left(\frac{1}{p^{+}}-\frac{\left(4 c_{1}\right)^{p^{-}} r T}{p^{-}}-A_{1} \varepsilon\left(\left.4 c_{1}\right|^{p^{-}}\right) \int_{0}^{T}|\dot{u}(t)|^{p(t)} d t-c_{6}\left(\int_{0}^{T}|\dot{u}(t)|^{p(t)} d t\right)^{\frac{\gamma+1}{p^{-}}}\right. \\
& -c_{7}\left(\int_{0}^{T}|\dot{u}(t)|^{p(t)} d t\right)^{\frac{1}{p^{-}}}+|\bar{u}|^{\gamma q}\left(\frac{1}{|\bar{u}|^{\gamma q}} \int_{0}^{T} F(t, \bar{u}) d t-A_{1} c(\varepsilon)\right)+c_{8}
\end{aligned}
$$

for all $u \in W_{T}^{1, p(t)}$ and a constant $c_{8}$, it follows from (iii) and Lemma $2.3, \varphi(u) \rightarrow+\infty$ as $\|u\| \rightarrow+\infty$. Applying Theorem 1.1 and Corollary 1.1 in Mawhin-Willem [19], we complete the proof.

Theorem 3.3 Let $F(t, x)=F_{1}(t, x)+F_{2}(x)$, where $F_{1}$ and $F_{2}$ satisfy $\left(F_{0}\right)$ and the following conditions:

(i) there exist $k(t), h(t) \in L^{1}\left([0, T] ; R^{+}\right)$and $\beta \in\left[0, p^{-}\right)$such that

$$
F_{1}(t, x) \geq-k(t)|x|^{\beta}+h(t)
$$

for all $x \in R^{N}$ and a.e. $t \in[0, T]$;

(ii) there exist constants $0 \leq r<\frac{p^{-}}{\left(4 c_{1}\right)^{p}} T_{p^{+}}$such that

$$
\left(\nabla F_{2}(x)-\nabla F_{2}(y), x-y\right) \geq-r|x-y|^{p^{-}}
$$

for all $x, y \in R^{N}$ and a.e. $t \in[0, T]$;

(iii)

$$
\begin{gathered}
\liminf _{|x| \rightarrow \infty} \frac{1}{|x|^{\beta}} \int_{0}^{T} F_{2}(x) d t \geq B \\
\text { as }|x| \rightarrow+\infty \text {, where } B=2^{\beta} \int_{0}^{T} k(t) d t .
\end{gathered}
$$

Then problem (1.1) has at least one nontrivial solution which minimizes $\varphi$ on $W_{T}^{1, p(t)}$.

Proof By condition (i) and Sobolev's inequality and Lemma 2.2, we get

$$
\int_{0}^{T} F_{1}(t, u(t)) d t \geq \int_{0}^{T}\left[-k(t)|u(t)|^{\beta}+h(t)\right] d t
$$




$$
\begin{aligned}
& \geq-2^{\beta}\left(|\bar{u}|^{\beta}+\|\tilde{u}(t)\|_{\infty}^{\beta}\right) \int_{0}^{T} k(t) d t+\int_{0}^{T} h(t) d t \\
& =-B|\bar{u}|^{\beta}-B\|\tilde{u}(t)\|_{\infty}^{\beta}+\int_{0}^{T} h(t) d t \\
& \geq-B\left(4 c_{1}\right)^{p_{-}}\left(\int_{0}^{T}|\dot{u}(t)|^{p(t)}\right)^{\frac{\beta}{p^{-}}}-B|\bar{u}|^{\beta}+c_{9}
\end{aligned}
$$

for all $u \in W_{T}^{1, p(t)}$ and a constant $c_{9}$, where $B=2^{\beta} \int_{0}^{T} k(t) d t$.

It follows from (3.6) and (3.8) that

$$
\begin{aligned}
\varphi(u)= & \int_{0}^{T} \frac{1}{p(t)}|\dot{u}(t)|^{p(t)} d t+\int_{0}^{T} F(t, u(t)) d t \\
\geq & \frac{1}{p^{+}} \int_{0}^{T}|\dot{u}(t)|^{p(t)} d t+\int_{0}^{T} F_{1}(t, u(t)) d t+\int_{0}^{T}\left(F_{2}(u)-F_{2}(\bar{u})\right) d t+\int_{0}^{T} F_{2}(\bar{u}) d t \\
\geq & \left(\frac{1}{p^{+}}-\frac{\left(4 c_{1}\right)^{p^{-}} r_{1} T}{p^{-}}\right) \int_{0}^{T}|\dot{u}(t)|^{p(t)} d t-B\left(4 c_{1}\right)^{p^{-}}\left(\int_{0}^{T}|\dot{u}(t)|^{p(t)}\right)^{\frac{\beta}{p^{-}}} \\
& +|\bar{u}|^{\beta}\left(\frac{1}{|\bar{u}|^{\beta}} \int_{0}^{T} F_{2}(\bar{u}) d t-B\right)+c_{10}
\end{aligned}
$$

for all $u \in W_{T}^{1, p(t)}$ and a constant $c_{10}$, it follows from (iii), $0<\beta<p^{-}, 0 \leq r<\frac{p^{-}}{\left(4 c_{1}\right)^{p^{-}} T p^{+}}$ and Lemma 2.3, $\varphi(u) \rightarrow+\infty$ as $\|u\| \rightarrow+\infty$. Applying Theorem 1.1 and Corollary 1.1 in Mawhin-Willem [19], we complete the proof.

\section{Examples}

In this section, we give some examples of $F(t, x)$ and $p(t)$ to illustrate our results.

Example 4.1 In system (1.1), let $p(t)=3+\cos \omega t, F(t, x)=F_{1}(t, x)+F_{2}(x)$, where

$$
\begin{array}{ll}
T=\frac{2 \pi}{\omega}, \quad p^{+}=4, & p^{-}=2, \quad \lambda=2, \quad \mu=3, \\
F_{1}(t, x)=|x|^{2}|\sin \omega t|, & F_{2}(x)=c(x)-\frac{1}{2} r|x|^{2},
\end{array}
$$

with

$$
r>0, \quad c(x)=\frac{1}{2} r\left(\left|x_{1}\right|^{3}+\left|x_{2}\right|^{2}+\left|x_{3}\right|^{2}+\cdots+\left|x_{N}\right|^{2}\right) .
$$

By some computation, we can take $0 \leq r<\frac{1}{32 c_{1}^{2} T}$ such that

$$
\left(\nabla F_{2}(x)-\nabla F_{2}(y), x-y\right) \geq-r|x-y|^{2}
$$

and $F_{1}(t, \cdot)$ is $(4,6)$-subconvex, for all $x, y \in R^{N}$ and a.e. $t \in[0, T]$.

It is easy to verify

$$
\frac{1}{\mu p^{-}} \int_{0}^{T} F_{1}\left(t, \lambda p^{-} x\right) d t+\int_{0}^{T} F_{2}(x) d t \rightarrow+\infty
$$

as $|x| \rightarrow+\infty$. 
Therefore all the conditions of Theorem 3.1 are satisfied, the problem (1.1) has at least one solution.

Example 4.2 In system (1.1), let $p(t)=5+\cos \omega t, F(t, x)=F_{1}(t, x)+F_{2}(x)$, where

$$
\begin{array}{ll}
\omega=\frac{2 \pi}{T}, \quad p^{+}=6, \quad p^{-}=4, & \gamma=\frac{3}{4}, \\
F_{1}(t, x)=|x|^{\frac{7}{4}}|\sin \omega t|+(h(t), x), & F_{2}(x)=c(x)-\frac{1}{4} r|x|^{4},
\end{array}
$$

with

$$
r>0, \quad h(t) \in L^{1}\left([0, T], R^{+}\right), \quad c(x)=\frac{1}{4} r\left(\left|x_{1}\right|^{6}+\left|x_{2}\right|^{4}+\left|x_{3}\right|^{4}+\cdots+\left|x_{N}\right|^{4}\right) .
$$

By some computation, we can take $0 \leq r \leq \frac{1}{32 c_{1}^{2} T}$ such that

$$
\begin{aligned}
& \left(\nabla F_{2}(x)-\nabla F_{2}(y), x-y\right) \geq-r|x-y|^{4}, \\
& \left|\nabla F_{1}(t, x)\right|=\frac{7}{4}|x|^{\frac{3}{4}}|\sin \omega t|+|h(t)|,
\end{aligned}
$$

for all $x, y \in R^{N}$ and a.e. $t \in[0, T]$.

On the other hand, we can verify that condition (iii) of Theorem 3.2 is satisfied. Therefore all the conditions of Theorem 3.2 hold, the problem (1.1) has at least one solution.

Example 4.3 In system (1.1), let $p(t)=\frac{5}{2}+\cos \omega t, F(t, x)=F_{1}(t, x)+F_{2}(x)$, where

$$
\begin{aligned}
& \omega=\frac{2 \pi}{T}, \quad p^{+}=\frac{7}{2}, \quad p^{-}=\frac{3}{2}, \quad \beta=\frac{1}{2}, \\
& F_{1}(t, x)=|x|^{\frac{1}{2}}\left(\frac{1}{2} \sin \omega t+1\right)+(h(t), x) \geq-|x|^{\frac{1}{2}} \frac{1}{2} \sin \omega t+(h(t), x), \\
& F_{2}(x)=c(x)-\frac{2}{3} r|x|^{\frac{3}{2}},
\end{aligned}
$$

with

$$
r>0, \quad h(t) \in L^{1}\left([0, T], R^{+}\right), \quad c(x)=\frac{2}{3} r\left(\left|x_{1}\right|^{2}+\left|x_{2}\right|^{\frac{3}{2}}+\left|x_{3}\right|^{\frac{3}{2}}+\cdots+\left|x_{N}\right|^{\frac{3}{2}}\right) .
$$

Similar to Example 4.1,we can see all the conditions of Theorem 3.3 are satisfied, the problem (1.1) has at least one solution.

\section{Acknowledgements}

We would like to thank the referee for his/her valuable comments and helpful suggestions which have led to an improvement of the presentation of this paper.

\section{Funding}

The authors were supported by NNSF of China (No. 11501165); the Fundamental Research Funds for the Central Universities (2018B58614). 
Competing interests

The authors declare that they have no competing interests.

\section{Authors' contributions}

All the authors contributed equally and significantly in writing this article. All the authors read and approved the final manuscript.

\section{Publisher's Note}

Springer Nature remains neutral with regard to jurisdictional claims in published maps and institutional affiliations.

Received: 28 February 2019 Accepted: 1 August 2019 Published online: 09 August 2019

\section{References}

1. Antontsev, S.N., Shmarev, S.I.: A model porous medium equation with variable exponent of nonlinearity: existence, uniqueness and localization properties of solutions. Nonlinear Anal. 60, 515-545 (2005)

2. Fan, X.L., Zhang, Q.H.: Existence of solutions for $p(x)$-Laplacian Dirichlet problems. Nonlinear Anal. 52, 1843-1852 (2003)

3. Avci, C.M.: Existence and multiplicity of solutions for Dirichlet problems involving the $p(x)$-Laplace operator. Electron. J. Differ. Equ. 14, 1 (2013)

4. Ayazoglu, R., Avci, M.: Positive periodic solutions of nonlinear differential equations system with nonstandard growth. Appl. Math. Lett. 43, 5-9 (2015)

5. An, Y.K., Ru, Y.F., Wang, F.L.: Existence of nonconstant periodic solutions for a class of second-order systems with p(t)-Laplacian. Bound. Value Probl. 2017, 170 (2017)

6. Rabinowitz, P.H.: Periodic solutions of Hamiltonian systems. Commun. Pure Appl. Math. 31, 157-184 (1978)

7. Zhikov, V.V.: Averaging of functionals of the calculus of variations and elasticity theory. Math. USSR, Izv. 9, 33-66 (1987)

8. Zhang, S.G.: Periodic solutions for a class of second order Hamiltonian systems with $p(t)$-Laplacian. Bound. Value Probl. 2016, $211(2016)$

9. Wang, Z.Y., Zhang, J.H., Zhang, Z.T.: Periodic solutions of second order non-autonomous Hamiltonian systems with local superquadratic potential. Nonlinear Anal. 70, 3672-3681 (2009)

10. Zhang, L., Tang, X.H., Chen, J.: Infinitely many periodic solutions for some differential systems with $p(t)$-Laplacian. Bound. Value Probl. 2011, 33 (2011)

11. Tan, Z., Fang, F.: On superlinear $p(x)$-Laplacian problems without Ambrosetti and Rabinowitz condition. Nonlinear Anal. 75, 3902-3915 (2012)

12. Zhang, L., Tang, X.H.: Existence and multiplicity of periodic solutions for some second order differential system with p(t)-Laplacian. Math. Slovaca 63(6), 1269-1290 (2013)

13. Zhang, L., Tang, X.H.: Periodic solutions for some nonautonomous $p(t)$-Laplacian hamiltontian systems. Appl. Math. 58(1), 39-61 (2013)

14. Edmunds, D.E., Rakosnik, J.: Sobolev embeddings with variable exponent. Math. Nachr. 246-247, $53-67$ (2002)

15. Fan, X.L., Zhao, D.: On the spaces $L^{p(x)}$ and $W^{m, p(x)}$. J. Math. Anal. Appl. 263, 424-446 (2001)

16. Fan, X.L., Shen, J.S., Zhao, D.: Sobolev embedding theorems for spaces $W^{k, p(x)}(\Omega)$. J. Math. Anal. Appl. 262, 749-760 $(2001)$

17. Fan, X.L., Zhao, Y.Z., Zhao, D.: Compact imbedding theorems with symmetry of Strauss-Lions type for the space $W^{1, p(x)}(\Omega)$. J. Math. Anal. Appl. 225, 333-348 (2001)

18. Wang, X.J., Yuan, R.: Existence of periodic solutions for $p(t)$-Laplacian systems. Nonlinear Anal. 70, 866-880 (2009)

19. Mawhin, J., Willem, M.: Critical Point Theory and Hamiltonian Systems. Springer, Berlin (1989)

20. Fan, X.L., Fan, X.: A Knobloch-type result for p(t)-Laplacian systems. J. Math. Anal. Appl. 282, 453-464 (2003)

21. Wu, X.P., Tang, C.L.: Periodic solution of a class of non-autonomous second order systems. J. Math. Anal. Appl. 236 227-235 (1999)

\section{Submit your manuscript to a SpringerOpen ${ }^{\odot}$ journal and benefit from:}

- Convenient online submission

- Rigorous peer review

- Open access: articles freely available online

- High visibility within the field

- Retaining the copyright to your article

Submit your next manuscript at $>$ springeropen.com 